\title{
Amplitude response of a unilaterally constrained nonlinear micromechanical resonator
}

\author{
Xueyong Wei ${ }^{1 \mathrm{a}}$, Federico Bizzarri ${ }^{\mathrm{b}}$, Carl Anthony ${ }^{\mathrm{c}}$, Mike CL Ward ${ }^{\mathrm{c}}$, David Lowe ${ }^{\mathrm{d}}$ \\ ${ }^{a}$ Department of Engineering, University of Cambridge, Trumpington Street,Cambridge, CB2 1PZ, UK \\ ${ }^{b}$ Dipartimento di Elettronica e Informazione (DEI),Politecnico di Milano, P.za. Leonardo da Vinci, n. 32, \\ I20133 Milano, Italy \\ ${ }^{c}$ School of Mechanical Engineering, University of Birmingham, Birmingham, B15 2TT, UK \\ ${ }^{d}$ Nonlinearity and Complexity Research Group (NCRG), Aston University, Birmingham, UK
}

\begin{abstract}
Dynamical systems which involve impacts frequently arise in engineering. This paper reports a study of such a system at micro scale that consists of a nonlinear resonator operating with an unilateral impact. The micro resonators were fabricated on SOI wafers by using a onemask process and then characterized by using the capacitively driving and sensing method. Numerical results concerning the dynamics of this vibro-impact system was verified by the experiments. Bifurcation analysis was used to provide a qualitative scenario of the system steady state solutions as a function of both the amplitude and the frequency of the external driving sinusoidal voltage. The results show that the amplitude of resonant peak is levelled off due to the impact effect and that the bandwidth of impacting is dependent upon the nonlinearity and the operating conditions.
\end{abstract}

Keywords: Microfabrication; Micromechanical resonator; Impact dynamics; Bifurcation analysis;

\section{Introduction}

Dynamical systems which involve impacts frequently arise in engineering. Although recurrent impacts need to be suppressed in many mechanical systems, they can also be utilized in various applications at micro and even nanoscale for the operation of devices. For example, a micromachined impact actuator (known as Mita's actuator) driven by electric forces was developed to produce a precise displacement [1]. A later theoretical study unveils the complex dynamics of Mita's actuator and provides a guideline for designing it [2, 3]. Another example is the atomic force microscopy (AFM) operating in the tapping mode. When the AFM cantiliver probe engages the surface of the sample under test, the mechanical nonlinearity and also the nonlinear interaction forces like van der walls forces have to be considered in the probe dynamics $[4,5]$.

In this paper, we report a study of such a dynamical system including an electrostatically driven nonlinear micro-mechanical resonator subject to unilateral impact. Our preliminary

\footnotetext{
${ }^{1}$ Email: xwei@asme.org
} 
studies $[6,7]$ shows that the nonlinearity can effectively changes the pull-in voltage for inducing the impact in the system, and more importantly, an autonomous oscillator based on the nonlinear resonator with impact shows a tunable frequency capability. In this follow on study, we successfully fabricated and characterized the devices. The rest of this paper is organized as follows. Sec.2 first describes the design concept and mathematical model of the proposed device operating as a nonlinear resonator with a unilateral rigid constraint. Then, the details of one-mask microfabrication process and open loop characterizations of the physcial system are given in Sec.3. The testing results are presented and discussed together with the numerical simulations in Sec.4. Final conclusions are given in Sec.5.

\section{Design and Modelling}

The designed micromechanical impact oscillator after microfabrication is shown in Fig.1a. The top electrode pad with a gold wire on it is used to capacitively drive the resonator through a parallel-plate structure (area of $A$ and gap of $g$ ) as shown in Fig.1b and the bottom electrode pad is used to sense the motion of the resonator through the comb finger structures. The frame connected with the plate acts as the resonator body supported by two folded beams and a doubly clamped beam. A dimple structure was designed on the top electrode as shown in Fig.1c to avoid stiction between the parallel-plates when a dynamic impact or pull-in takes place during operation.

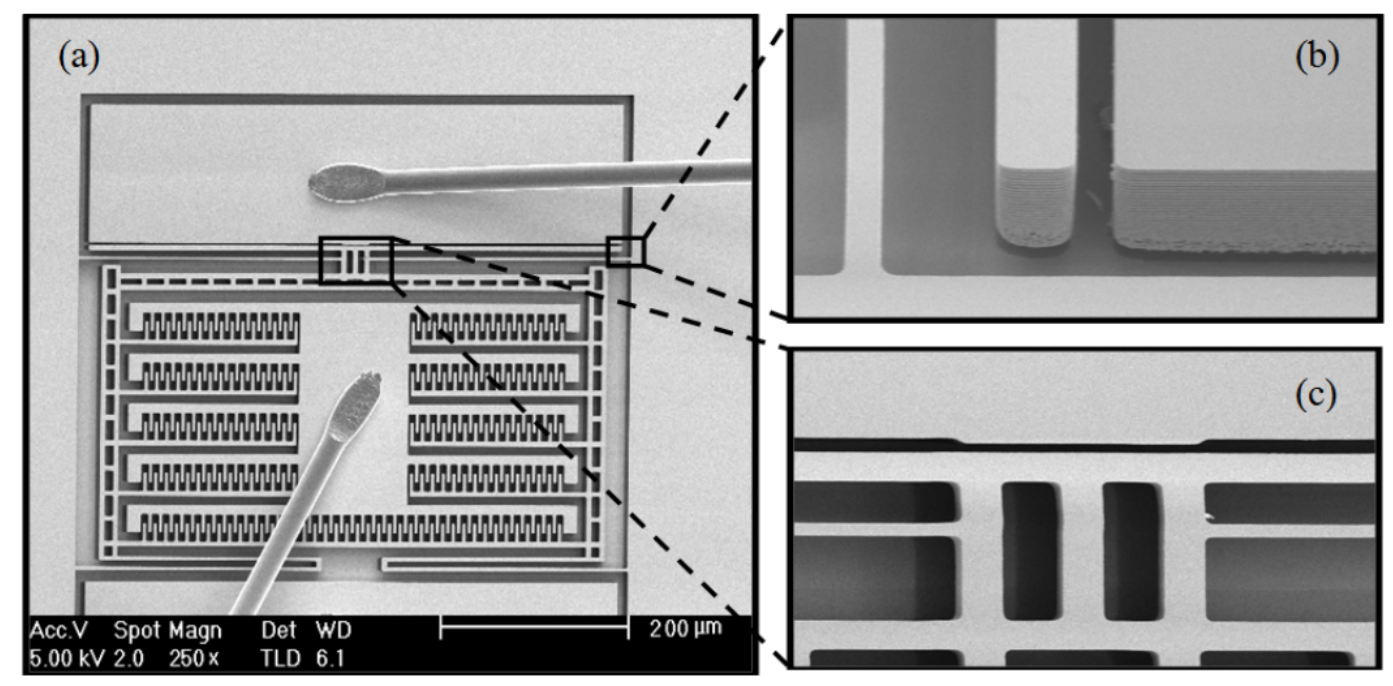

Figure 1: SEM micrograph of the microfabricated device. The wire on the top pad is used to excite the resonator and the one on the bottom pad is used to sense the motion of the resonator.

Once an alternative electrical voltage is applied between the parallel plates, the resonator starts to oscillate around its equilibrium point due the electrostatic force and the equation of motion can be written as: 


$$
m \ddot{z}+c \dot{z}+k_{1} z+k_{2} z^{2}+k_{3} z^{3}=F_{e}(z, t),
$$

where $z$ is the displacement of the resonator, $m$ is the lumped mass, $c$ is the damping coefficient, $k_{1}, k_{2}$ and $k_{3}$ are the effective linear, quadratic and cubic spring constants respectively. The applied electrostatic force $F_{e}(z, t)$ can be expressed as

$$
F_{e}(z, t)=\frac{1}{2} \frac{d C_{d}}{d z}\left(V_{d c}+V_{a c} \sin \left(2 \pi f_{d} t\right)\right)^{2}+\frac{1}{2} \frac{d C_{s}}{d z} V_{s}^{2}
$$

where $\frac{d C_{d}}{d z}=\frac{\epsilon A}{(g-z)^{2}}$ is the capacitance gradient of the parallel plate and $\frac{d C_{s}}{d z}$ is that of the comb finger structures. $V_{d c}$ and $V_{s}$ are the bias voltages applied on the driving and sensing electrodes. $V_{a c}$ and $f_{d}$ are the amplitude and frequency of the applied alternate current (AC) driving voltage. For both numerical simulations and experimental results reported in Sec.3, $V_{d c}$ is set to zero and $f_{d}$ is accordingly varied around sub-harmonic frequency $\left(f_{0} / 2\right)$.

Due to the dimple structure on the top electrode, the maximum value of $z$ is unilaterally limited by the local gap distance $d$. Therefore, Eq. 1 is only valid when $z<d$. When $z \geq d$, the resonator collides with the dimple. The impact can be modelled by a simple elastic collision and is described as follows.

$$
\dot{z}^{+}\left(t_{I}\right)=-r \dot{z}^{-}\left(t_{I}\right)
$$

Where, (i) "-" and "+" are the subscripts chosen to represent variables before and after an impact takes place (say at $t=t_{I}$ ), and (ii) $r \in[0,1]$ the coefficient of restitution, whose value depends upon the material properties of the contacting objects and the dynamics of the collision. For the sake of simplicity, $r$ is set as 1 in the numerical simulation of this work.

Before electrical testing, the designed devices were first simulated using FEA package. Key parameters from the design and simulation are given in Table 1 . The dynamical behavior was further investigated by solving the governing equations and the results are discussed in Sec.4.

Table 1: Key Parameters from Design and FEA Simulation

\begin{tabular}{c||c||c}
\hline Parameter & Value & Units \\
\hline \hline$m$ & $1.808 \cdot 10^{-9}$ & $\mathrm{~kg}$ \\
$k_{1}$ & 7.567 & $\mathrm{~kg} \mathrm{sec}^{-2}$ \\
$k_{2}$ & $1.894 \cdot 10^{4}$ & $\mathrm{~kg} \mathrm{sec}^{-2}$ \\
$k_{3}$ & $3.206 \cdot 10^{11}$ & $\mathrm{~kg} \mathrm{~m}^{-2} \mathrm{sec}^{-2}$ \\
$A$ & $1.2 \cdot 10^{-8}$ & $\mathrm{~m}^{2}$ \\
$g$ & $2.5 \cdot 10^{-6}$ & $\mathrm{~m}$ \\
$d$ & $1.5 \cdot 10^{-6}$ & $\mathrm{~m}$ \\
$\frac{d C_{s}}{d z}$ & $1.503 \cdot 10^{-8}$ & $\mathrm{~F} \mathrm{~m}^{-1}$ \\
$\epsilon_{0}$ & $8.85 \cdot 10^{-12}$ & $\mathrm{~F} \mathrm{~m}^{-1}$ \\
\hline
\end{tabular}




\section{Experimental details}

\subsection{Microfabrication of the device}

The micro device reported in this paper was fabricated on silicon on insulator (SOI) wafers of $20 \mu \mathrm{m}$ thick device layer, $2 \mu \mathrm{m}$ thick intermediate $\mathrm{SiO}_{2}$ layer and $500 \mu \mathrm{m}$ thick handling layer. The SOI wafers were first lithographically patterned by using S1805 positive photoresist and then resonator patterns were transferred into the silicon layer through a deep reactive ion etching (DRIE) process. After the DRIE, the wafer was diced into individual chips and an underetch of the sacrificial layer was conducted in the chip form by using a $\mathrm{HF}$ vapor etching for 40 mins at $35{ }^{\circ} \mathrm{C}$. The released resonators were metalized with a thin $\mathrm{Au} / \mathrm{Cr}$ layer of 200nm thick and annealed in nitrogen gas to form a bondable ohmic contact. The fabricated device is shown in Fig.1. Thae SEM measured distances from the moving plate to the fixed one $(\mathrm{g})$ and to the bumper $(\mathrm{d})$ are respectively $2.41 \mu \mathrm{m}$ and $1.46 \mu \mathrm{m}$, which are less than the designed values of $2.5 \mu \mathrm{m}$ and $1.5 \mu \mathrm{m}$ due to microfabrication errors.

\subsection{Open-Loop Characterization}

Micromachined resonators operating in air normally show a degraded performance because the fluid damping related energy loss is significant. Our fabricated devices were accordingly tested in a customized chamber and the measurement setup for characterizing their dynamics is shown in Fig.2. Compared with optical detection scheme, capacitive readout method is much easier to implement, and both the driving and sensing of the device can be electrically controlled. In the experiment, a harmonic voltage $\left(V_{i n}=V_{d c}+V_{a c} \sin \left(2 \pi f_{d} t\right)\right)$ was applied on the driving electrode (DE) to generate the electrostatic force to drive the resonator into oscillation. A DC sense voltage $V_{s}(8 \mathrm{~V})$ was applied on the readout combs, the moving resonator changes the capacitance and accordingly developes a motional current $\left(i_{m}\right)$ proportional to the instantaneous velocity given as,

$$
i_{m}=V_{s} \frac{d C}{d z} \frac{d z}{d t} .
$$

The induced motional current picked up through the sensing eletrode (SE) is then converted to voltage through LMC6084 operational amplifier on the interfacing circuit board. The voltage signal is captured by a data acquisition system (DAQ, National Instruments PCI-6133) with maximum sampling rate of $2.5 \mathrm{MSamples} / \mathrm{s}$. The data is further processed to analyse the dynamics of the device and the results are given in Sec.4.

\section{Results and discussions}

Fig.3(a) shows the simulation results of the resonator's amplitude response under different driving voltage $V_{a c}$. Based on the physical model and parameters in Sec.2, a critical value of $V_{a c}$ that is $V_{a c}^{c}=0.946 \mathrm{~V}$ was found from the numerical simulation. For $V_{a c}<V_{a c}^{c}$, the forced resonator oscillates within the allowed displacement range, in other words, there is no impact and Eq.1 holds. The frequency response curve (FRC) bends towards the higher frequency side and shows an abrupt jumping to lower amplitude due to the frequency hysteresis. According to Landu [8], the amplitude-dependent resonant frequency is expressed as: 


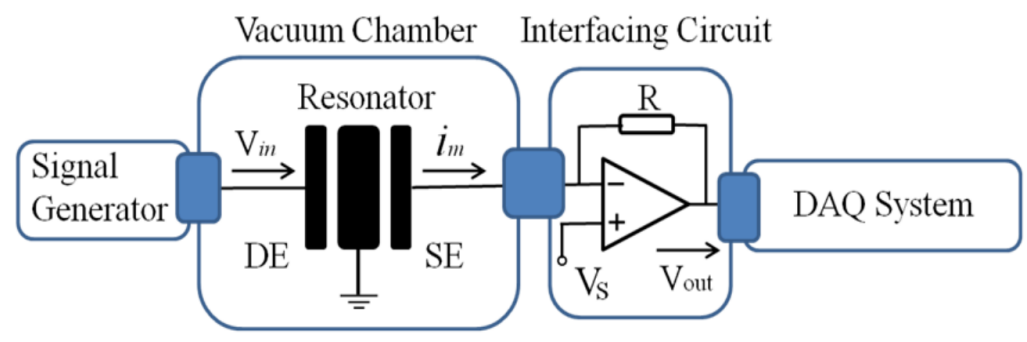

Figure 2: Schematic drawing of the measurement setup for testing the resonator.

$$
f_{0}^{\prime}=f_{0}+\kappa Y_{0, f_{0}^{\prime}}^{2}
$$

where $Y_{0, f_{0}^{\prime}}$ is the vibration amplitude at $f_{0}^{\prime}$ and $\kappa$ is a nonlinear parameter determined by the nonlinear spring constants:

$$
\kappa=f_{0}\left(\frac{3 k_{3}}{8 k_{1}}-\frac{5 k_{2}^{2}}{12 k_{1}^{2}}\right)
$$

For $V_{a c} \geq V_{a c}^{c}$, the unilateral bumper constraints the resonator's amplitude and accordingly the FRC starts to level off as shown in the inset of Fig.3(a). The starting point $P_{1}$ of on the FRC is a special point, at which the resonator just touches the bumper with a zero velocity. The system shows the dynamical behavior of a nonlinear resonator before $P_{1}$, while it becomes different due to the existence of grazing point. In order to have a complete picture of the dynamical behaviours in the parameter space of interest, bifurcation analysis of this periodically-forced nonlinear micro impact oscillator was carried out by resorting to the TC-HAT continuation toolbox [9] for hybrid systems. The parameter space of interest is formed by the driving signal parameters $V_{a c}$ in $[0,4]$ and $f_{d}$ in $\left[0.475 f_{0}, 0.55 f_{0}\right]$. As shown in Fig.3(b), the $\left(f_{d} / f_{0}, V_{a c}\right)$ plane is divided into four regions $R_{i}$ where $i=1, \ldots, 4$ showing different dynamical behaviours. It is indicated that the system shows a typical saddle-node bifurcation if there is no impacting occurs and the plane is separated by the limit-cycle bifurcation curve $L P C_{a}$ and $L P C_{b}$ into $R_{1}$ where a stable limit cycle motion dominates and $R_{2}$ where two stable limit cycles coexist with an unstable one. As demonstrated in the experiment, the grazing phenomenon occurs once the driving amplitude is increased big enough at the right tone. Therefore, the grazing bifurcation curve $G R$ further divides $R_{2}$ into three $R_{2}, R_{3}$ and $R_{4}$. In $R_{3}$, a stable impacting limit cycle coexists with both a stable and an unstable non-impacting one. It is important to notice that the point $Q$ on $G R$ is a special point since it is the origin of both $L P C_{a}$ and $L P C_{b}$ on $G R$. A further grazing bifurcation occurring on the right branch of $G R$ is responsible for the unstable impacting limit cycle coexisting in $R_{4}$ with both a non impacting and an impacting stable one.

As expected from the numerical simulation, the experimental measured frequency responses plotted in Fig.4(a) show a similar trend. According to Eq.6, $f_{0}=14103 \mathrm{~Hz}$ and $\kappa=1.224 \times 10^{14} \mathrm{~Hz} / \mathrm{m}^{2}$ are extracted from the measured resonant peaks $P_{1}$ and $P_{2}$ on the FRCs in Fig.4(a). The discrepancy between the designed and measured system parameters 


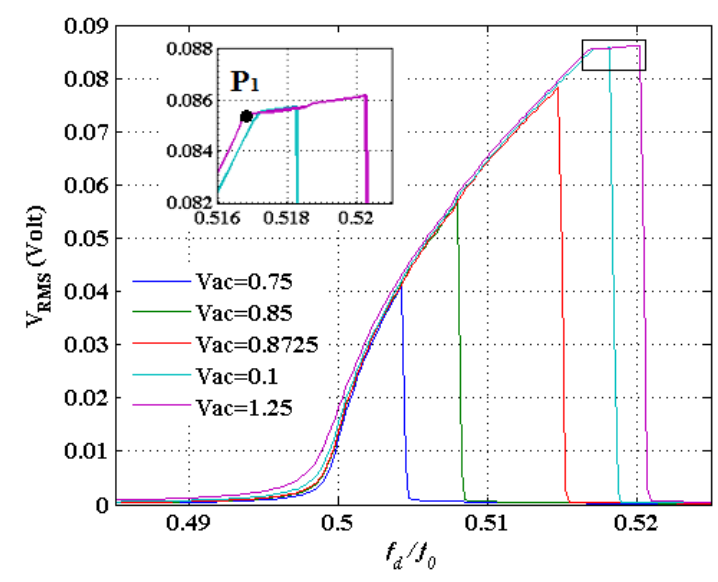

(a)

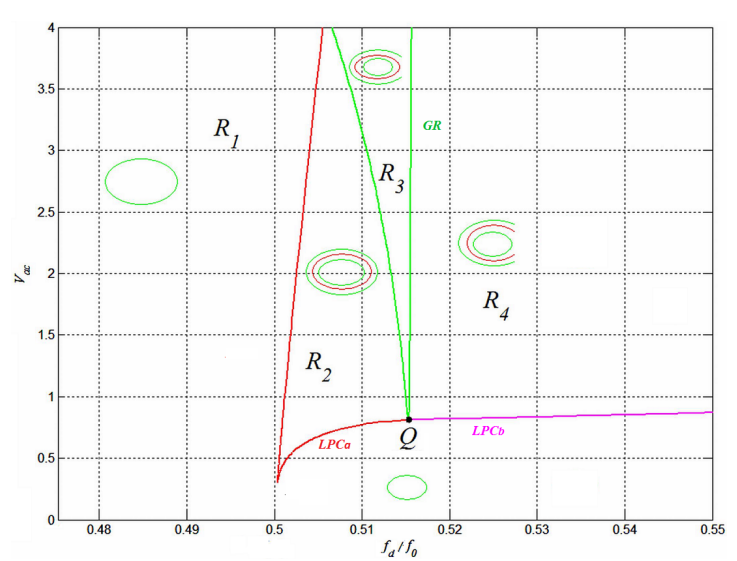

(b)

Figure 3: (a)Simulation results of the resonator reponse to numerical frequency sweeping tests (b)Bifurcation diagram of this hybrid system

like resonant frequency and stiffness is because of the manufacturing variation in our microfabrication process, which has been discussed in our previous work [10]. Interestingly, however, it was found from experiment that the output voltage of frequency response has a saturation value $V_{\text {out }}^{c}$ of $71.6 \mathrm{mV}$. On the FRC of $V_{a c}=1.5 \mathrm{~V}$ as shown in Fig.4(a), the impacting region is labled as $\overline{P_{3} P_{4} P_{5}}$, where $P_{3}$ is a turning point at which the amplitude gradient is dramatically decreased. After $P_{3}$, the output voltage continues to increase slowly up to $V_{\text {out }}^{c}$ at $P_{4}$ and remains almost constant until the jumping point at $P_{5}$. As indicated in Eq.6, the bigger $\kappa$ and $V_{a c}$ are, the larger bandwidth of impact curve $\overline{P_{3} P_{4} P_{5}}$ is. To further investigate the saturation behavior of the output signal, an amplitude sweeping test was carried out by fixing the driving frequency $f_{d}$ during each run of sweeping and $V_{a c}$ was increased from $0.25 \mathrm{~V}$ to $2.5 \mathrm{~V}$ with a step of $0.05 \mathrm{~V}$ illustrated by the sweeping path (SWP1, SWP2 and SWP3) in Fig.4(a). The results are shown in Fig.4(b). Take $f_{d}=7050 \mathrm{~Hz}$ for example, the resonator operates on its upper branch of FRC and its output grows smootly following SWP1 as $V_{a c}$ increases. When $V_{a c}$ reaches $1.9 \mathrm{~V}$, the resonator grazes the bumper at $P_{3}$ with an output voltage of $V_{\text {out }}=65 \mathrm{mV}$. After $P_{3}$, the resonator collides with the bumper, but the increase of output voltage continues until it approaches the saturation point at $P_{4}$ with $V_{\text {out }}=71.6 \mathrm{mV}$. Compared with the results of frequency sweeping test shown in Fig.4(a), one can conclude that there is a saturated output voltage of this nonlinear micro impact oscillator. It should be noted that, however, the path to approach the saturation point is not necessary to be continuous. Take SWP2 for example, the resonator operates on the lower branch of FRC at $f_{d}=7090 \mathrm{~Hz}$. As $V_{a c}$ increases, the bifurcation point shifts to the right and a nonlinear jumping takes place at $V_{a c}=1.55 \mathrm{~V}$ from $P_{1}$ up to $P_{2}$ at the upper branch. Accordingly, the output voltage reaches the saturation value immediately and remains constant after $P_{2}$. Therefore, the selection of $f_{d}$ determines the evolution of dynamic behaivor of the nonlinear resonator during the amplitude sweeping. For the resonator operates on its lower branch of FRC where $f_{d}=7125 \mathrm{~Hz}$ (SWP3), the output amplitude grows slowly as expected and a much larger $V_{a c}$ needs to be applied in order to reach the staturation 
point. The saturation of output signal indicates that there is a varying contact loss or other mechanism to compensate the over pumped energy. This can be partially explained by the non-ideal elastic collision because the microfabricated device shows a typical scalloped sidewall as shown in Fig.1b and the sidewall surface is not perfectly flat as assumed.

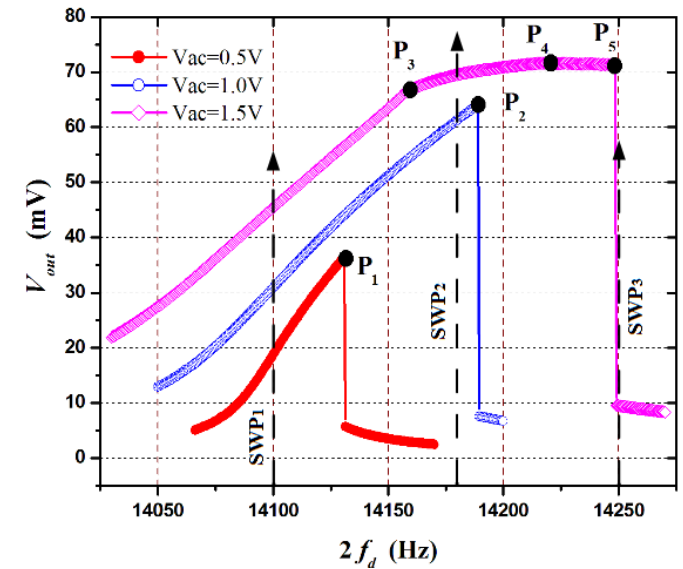

(a)

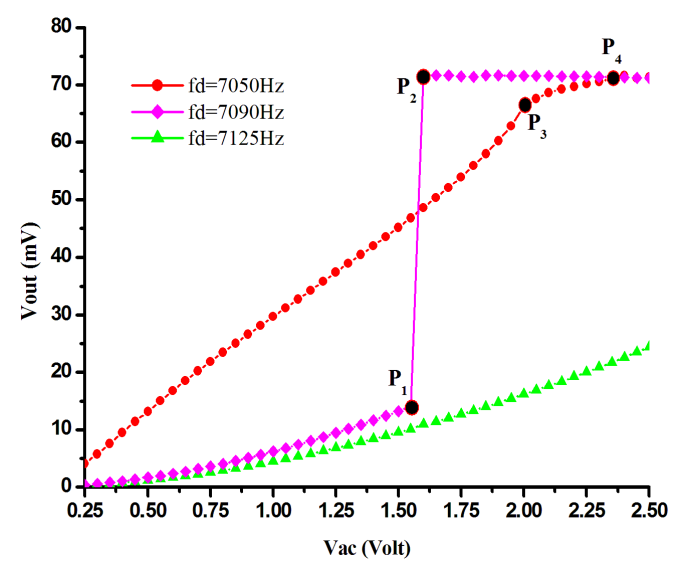

(b)

Figure 4: (a)Experimental results of the resonator response to frequency sweeping tests (b)Resonator's output from the amplitude sweeping tests with driving frequency fixed in each run

\section{Conclusions}

In this paper, a follow on study of the previously introduced unilaterally constrained nonlinear micromechanical resonator is reported. This in-plane vibration based devices were successfully fabricated using our one mask SOI process. The simulation and testing results show that the nonlinear resonant peak is leveled off due to the unilateral constraint and the bandwidth of levelled curve is dependent on the nonlinearity and the operating conditions. Different from simulation, a saturation of the output signal was found in the experiment, indicating that there could be a varying contact loss due to the non-ideal elastic impact. The analysis shows that the system under periodical driving can lead to complex dynamics when grazing bifurcation is considered.

\section{Acknowledgment}

The authors acknowledge the support from the Engineering and Physical Sciences Research Council (EPSRC) and the State Key Laboratory of Mechanical System and Vibration, Shanghai Jiaotong University (MSV-2010-20). The Authors would like to thanks Dr. Phanikrishna Thota for his help concerning the use of the continuation toolbox TC-HAT. 


\section{References}

[1] M. Mita, M. Arai, S. Tensaka, D. Kobayashi, H. Fujita, Amicromachined impact microactuator driven by electrostatic force, IEEE Journal of Microelectromechanical Systems 12 (2003) 37-41.

[2] X. Zhao, H. Dankowicz, C. Reddy, A. Nayfeh, Modeling and simulation methodology for impact microactuators, J. Micromech. Microeng. 14 (2004) 775-784.

[3] X. Zhao, C. Reddy, A. Nayfeh, Nonlinear dynamics of an electrically driven impact microactuator, Nonlinear Dyn 40 (2005) 227-239.

[4] A. S. Paulo, R. Garcia, Tip-surface forces,amplitude, and energy dissipation in amplitude-modulation (tapping mode) force microscopy, Phys. Rev. B 64 (2001) 193411.

[5] S. Lee, S. Howell, A. Raman, R. Reifenberger, Nonlinear dynamics of microcantilevers in tapping mode atomic force microscopy: A comparison between theory and experiment, Phys. Rev. B 66 (2002) 115409.

[6] X. Wei, C. Anthony, D. Lowe, M. CL.Ward, Design and fabrication of a nonlinear micro impact oscillator, Procedia Chemistry 1 (2009) 855-858.

[7] F. Bizzarri, X. Wei, Phase noise analysis of a mechanical autonomous impact oscillator with a mems resonator, in: Proceeding of 20th European Conference on Circuit Theory and Design (ECCTD), 2011, pp. $729-732$.

[8] L. Landau, E. Lifshitz, Mechanics 3rd edn, Oxford, UK: Butterworth-Heinemann, 1999.

[9] P. Thota, H. Dankowicz, Tc-hat $(\widehat{T C})$ : A novel toolbox for the continuation of periodic trajectories in hybrid dynamical systems, SIAM J. on Applied Dynamical Systems 7 (2008) 1283-1322.

[10] X. Wei, N. H. Saad, M. CL.Ward, Analysis of manufacturing variation in a coupled microresonators array based on its designed values and measured eigenfrequencies, Micro. Nano. Lett. 5 (2010) 855-858. 